\title{
A Novel Causal Model Based Distributed Fault Detection Method For Locomotive Electric Traction System
}

\author{
Chengshu, Chen Te-fang, Liwei \\ College of Information Science and Technology \\ Engineering \\ Central South University \\ Changsha, China \\ 6409020@qq.com
}

\author{
Ding Rong-jun \\ CSR Zhuzhou Institute Co. Ltd. \\ Zhuzhou, China \\ dingrj@teg.cn
}

\begin{abstract}
Aimed on the massive computation burden, lack of precise fault mathematic model and uncertainty of analysis method on subsystem parasitic parameters, a novel distributed fault diagnosis Method based on causal Model has been proposed. By the way of splitting entire system into several causal independent subsystems, the fault diagnosis can be divided, thus proper diagnosis method can be applied to each subsystem individually, without consideration of system frame and parameters. This Method lowers the complexity of diagnosis so as to enhance the reliability. By using this method, different diagnosis technology can be used on each subsystem; the accuracy of diagnosis has also been improved. In addition, this method can diagnosis pseudo-fault phenomenon caused by the coupling relationship between subsystems. The prototype has been applied to electrical locomotive HXD30132I .
\end{abstract}

Keywords- distributed system; fault diagnosis; causal Model; locomotive electric traction system;

\section{INTRODUCTION}

Electric traction system is one of the most important subsystems of electric locomotives. As an energy supplier for electric locomotives, Electric traction systems' failures will directly lead serious operational accidents, like locomotive failure. The safety of railway has won attention increasingly. How to make monitoring of real-time status and fault diagnosis of electric traction system work are the problems that must be solved to ensure the safety of railway operation immediately.

Electric traction system includes several subsystems, such as transformer, converter, driver, controller, among which, there is complicated coupling relationship. The traditional way of diagnosis is hard to be effective to diagnose faults of electric traction system ${ }^{[2]}$.

\section{BASIC IDEA OF DISTRIBUTED DIAGNOSIS AND ITS PRESENT SITUATIONS AT HOME AND ABROAD}

This paper presents a new thinking using causal model to perform distributed fault diagnose for fault diagnosing accurately and efficiently on industrial systems consisted by a set of complicated and strongly coupled subsystems. On one hand, the whole system is decomposed to several independent subsystems by analyzing system causal model and researching on partitioning algorithm to make every subsystem independent relatively. This will enable the function of fault diagnosis to be proceeded on parts of every system. On the other hand, by which the complexity and the amount of computation are reduced to design the most suitable diagnose to improved the accuracy of partial diagnose based on the structure and feature of candidates.

At present, common distributed fault diagnosis method need to split target system into subsystems; algorithm that calculating the regularized partition under given partition conditions is being introduced in article [3], this algorithm aims to system partition which need inter-regional information exchanging of common variables, there are similar method mentioned in article [4] and [5]. Disadvantages of single diagnosis method on complicated systems is emphasized in article [6] and [7], while a ideal of combining various diagnosis method to deal with complicated system are proposed. The global diagnosis results is optimizing through optimizing local diagnosis results. Although above method shows measurable improvement in local diagnosis effects, it's still hard to ensure the relative independence of local diagnosis, due to the lack of a practical and effective partition method, as a result, there is no assurance that the communication is minimized among subsystems while obtaining global diagnosis. This paper presents a partition algorithm based on causal model that can ensure the independence of partial diagnosis on subsystems and the communication minimization among systems based on further research of the structure and the causes for the breakdown of electric traction system.

\section{THE PROPOSED DISTRIBUTE FAULT CAUSAL MODEL}

Here, the electric traction system is taken for an example, to describe the basic principle of distributed fault diagnosis method based on causal model.

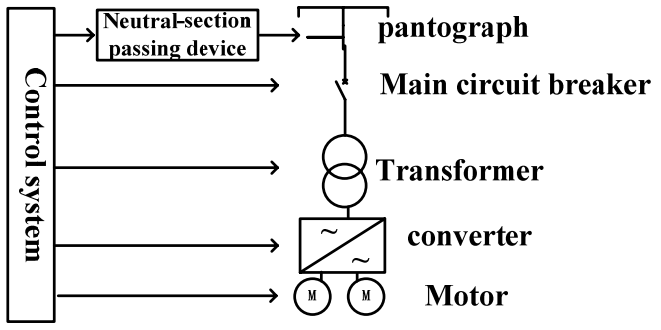

Figure 1. Topology of main circuit 
With complicated structure, electric traction system has more failures than other parts in electric locomotives. Fault statistical data of some certain electric locomotive in some certain local railway bureau of China in 2012 said that key electric parts with higher failure rate include pantograph, main circuit breaker, Neutral-section passing device, transformer, converter, motor and control system. Basically, simplified schematic circuit diagram of electric traction system at present is above.

The fault transfer pathway is just like the way how the electrical energy is turned to kinetic energy through pantograph, main circuit breaker, transformer, converter, motor to implement traction. TABLE I shows how electric traction system of electric locomotives is divided into subsystems with the source of trouble. Fig.2 shows the mutual influence among the subsystems analyzed and simplified by the analysis of vast data obtained at site and the statistics of fault records.

TABLE I. FAULT SOURCE PARTS

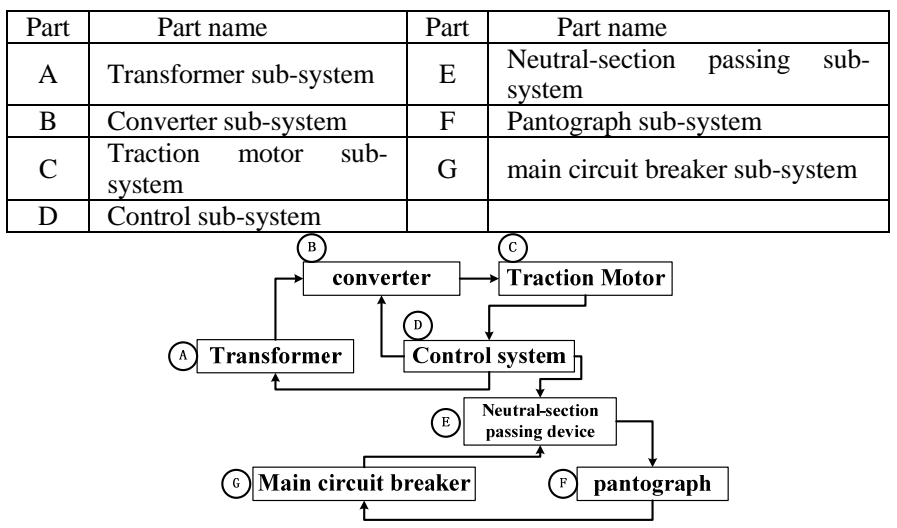

Figure 2. fault causal model of electric traction system

\section{THE RESEARCH ON PARTITION METHOD FOR PROPOSED FAULT CAUSAL MODEL}

In last section, a causal model of electric traction system fault is presents based on the analysis of the data obtained at site. To further analyze the fault causal model, 3 steps have to be done as follow: 1、Classification the causal model and assign the feedback loop into different levels obtained by model classification.2、Transform the target cyclic causal model into corresponding acyclic causal model.3、 Built up independent partition of obtained acyclic causal model to ensure effectiveness and independence of the partition.

The proposed fault causal model of the system is split into a set of respectively independent fault area by above steps. Partition structure achieved is optimized to make partial diagnose easy and efficient and global one accurate.

\section{A. Classification of causal model}

To simplify the classification procedure, several definitions are made. Reachable set of $\mathbf{V}_{\mathbf{i}}\left(\mathbf{R}_{\mathbf{i}}\right)$ : the vertex set that all the vertex in this set can be reached from vertex $V_{i}$ directly. Adjacency set of $\mathbf{V}_{\mathbf{i}}\left(\mathbf{A}_{\mathbf{i}}\right)$ : the vertex set that all the vertex in this set can reach $\mathrm{V}_{\mathrm{i}}$ directly. The classification rule is designed as the following equation (1):

$$
\begin{aligned}
& R_{i}=\left\{v_{i} \in V: m_{i j}=1\right\} \\
& A_{i}=\left\{v_{j} \in V: m_{j i}=1\right\} \\
& L_{1}=\left\{v_{i}: R_{i} \cap A_{i}=A_{i}\right\} \\
& L_{j}=\left\{v_{i}: v_{i} \in\left(V-L_{1}-\ldots-L_{j-1}\right) \quad\right. \text { and } \\
& \left.\left(R_{i}-L_{1}-\ldots-L_{j-1}\right) \cap\left(A_{i}-L_{1}-\ldots-L_{j-1}\right)\right\}, \\
& \text { where }: j=2, \ldots, m
\end{aligned}
$$

The system in fig 2 has a reachable matrix $\mathrm{M}$ as follow:

TABLE II. REACHABLE MATRIX

\begin{tabular}{|c|c|c|c|c|c|c|c|}
\hline & $\mathbf{A}$ & $\mathbf{B}$ & $\mathbf{C}$ & $\mathbf{D}$ & $\mathbf{E}$ & $\mathbf{F}$ & $\mathbf{G}$ \\
\hline $\mathbf{A}$ & 1 & 1 & 1 & 1 & 1 & 1 & 1 \\
\hline $\mathbf{B}$ & 1 & 1 & 1 & 1 & 1 & 1 & 1 \\
\hline $\mathbf{C}$ & 1 & 1 & 1 & 1 & 1 & 1 & 1 \\
\hline $\mathbf{D}$ & 1 & 1 & 1 & 1 & 1 & 1 & 1 \\
\hline $\mathbf{E}$ & 0 & 0 & 0 & 0 & 1 & 1 & 1 \\
\hline $\mathbf{F}$ & 0 & 0 & 0 & 0 & 1 & 1 & 1 \\
\hline $\mathbf{G}$ & 0 & 0 & 0 & 0 & 1 & 1 & 1 \\
\hline
\end{tabular}

The classification of fault causal model of electric traction system in fig.2 can be calculated by using equation (1) and table II as follow:

$$
\begin{aligned}
\forall U \in\{A, B, C, D\}: \mathrm{R}_{\mathrm{U}} \cap \mathrm{A}_{\mathrm{U}}=\mathrm{A}_{U} \\
\Rightarrow \mathrm{L}_{1}=\{\mathrm{A}, \mathrm{B}, \mathrm{C}, \mathrm{D}\} \\
\forall \mathrm{V} \in\{A, B, C, D\}:\left(\mathrm{R}_{\mathrm{V}}-L_{1}\right) \cap\left(\mathrm{A}_{\mathrm{V}}-L_{1}\right)=\left(\mathrm{A}_{V}-L_{1}\right) \\
\Rightarrow L_{2}=\{E, F, G\}
\end{aligned}
$$

\section{B. The minimize of feedback loop edge cut set}

Every level has only one strongly connected component (SCC), and this SCC set contained the complete feedback structure of the intact system causal model. So, the edge cut set of SCC is the only missing part to break the feedback loop without losing system details, the edge cut set of SCC has to be minimized while cut all the feedback loop structures. The Breadth-first search method (BFS) is adopted to search the minimized edge cut set (MECS) of SCC. Here a MECS searching algorithm is proposed:

\section{Algorithm 1: MECS searching}

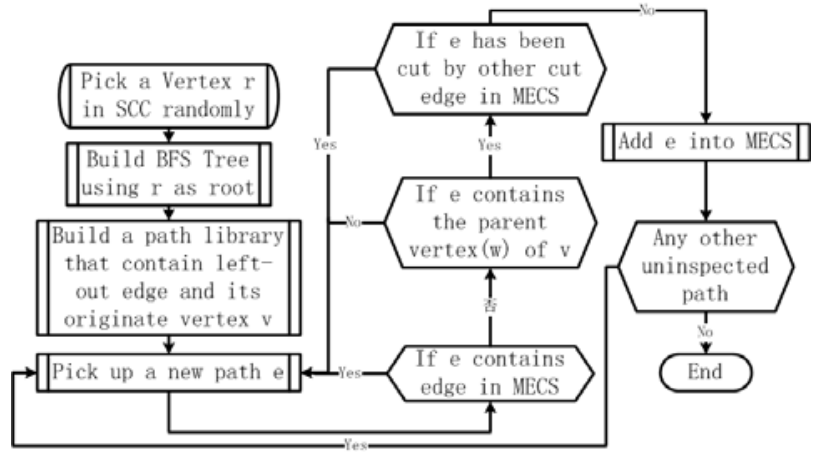

Figure 3. the procedure of algorithm 1

Where, Left-out edge is an edge that does not belong to the BFS tree. 


\section{Corresponding acyclic causal model}

Based on the above analyzing, feedback loop unfolding algorithm ${ }^{[8-9]}$ in time domain can be used to transfer target cyclic causal model into corresponding acyclic causal model, define $\mathrm{C}_{\max }$ as the time-window span constant. The procedure of algorithm 2 is designed below:

Algorithm 2: Feedback loops replacement

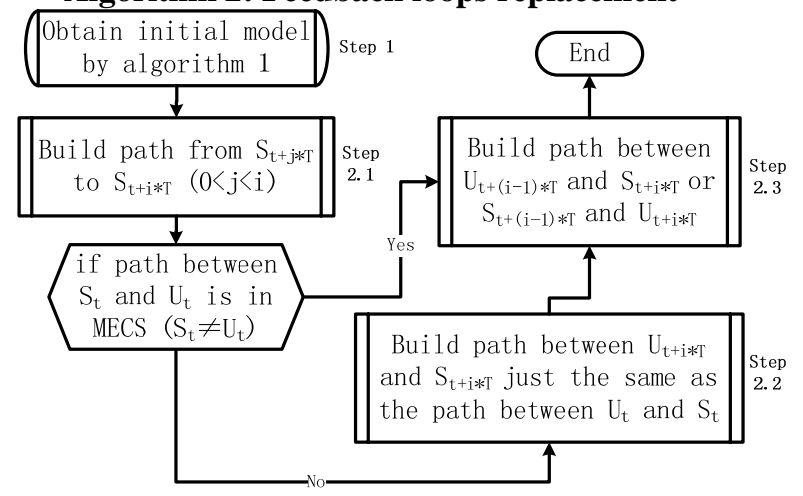

Figure 4. the procedure of algorithm 2

Where $S_{t}$ is an element of obtained initial model, and its instance at i-th time-step is noted as $\mathrm{S}_{\mathrm{t}+\mathrm{i} * \mathrm{~T}}$. $\mathrm{U}_{\mathrm{t}}$ is another element of the model that is not equal to $\mathrm{S}_{\mathrm{t}}$.

\section{A proposed partitioning method for acyclic causal model}

After obtained corresponding acyclic causal model of the system, a novel algorithm is present in this section to perform partitioning of the directed graph. This algorithm can ensure 2 goals that (1) the obtained partition has the minimal cut edge set and (2) the adjacent regions are causally independent. The procedure of algorithm 3 is designed as below, and here is a theorem been proven before that each hyper edge-cut set in HG has a correspondent vertex-cut set in MG of the same size. ${ }^{[10]}$

\section{Algorithm 3: Partitioning algorithm}

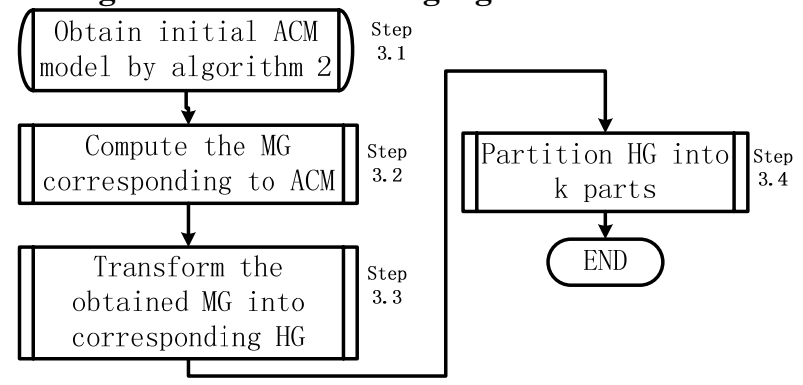

Figure 5. the procedure of algorithm 3

Where ACM is acyclic causal model, MG is moral graph and HG stands for hyper-graph.

The transfer process in step 3.3 obey the following rules that (1) node sets obtained by partitioning in MG correspond to the hyper edge-cut sets of HG and (2) The node sets in MG partitioning are delimited by edge labeling of MG provided by the HG partition, So the cut edge set of MG partition ACM into causally independent regions.
There are two constant has to be chosen by user during the HG partitioning process (step3.4). $\mathrm{k}$, which indicates the number of size balanced regions, and c, which indicates the overall imbalance tolerance between different regions.

\section{E. Application of proposed partitioning algorithm in electric traction system}

This section presents the application of the proposed algorithms on causal model of electric traction system in fig.2; the causal model is partitioned into causally independent regions. The initial model of directed graph in fig. 2 is shown in fig. 6 .

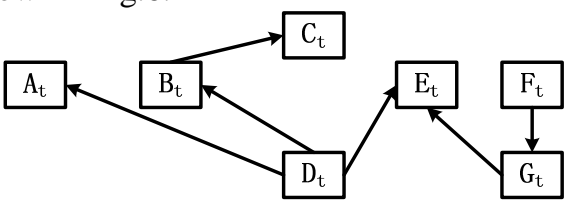

Figure 6. the initial model of system in fig.2

Utilize algorithm 1 to calculate the MECS of the each level obtained by classification of causal model. Cause any edge connect node $\mathrm{E}, \mathrm{F}, \mathrm{G}$ can be the minimal edge cut set for level 2, E $\rightarrow \mathrm{F}$ is selected as its MECS randomly. The time constant $\mathrm{c}$ is chosen to be 1 , and then algorithm 2 is applied to obtain the ACM corresponding to the electric traction system's fault causal model, which is shown in fig.7. Where the dotted line is produced by step 2.1 and the dashed line is produced by step 2.3

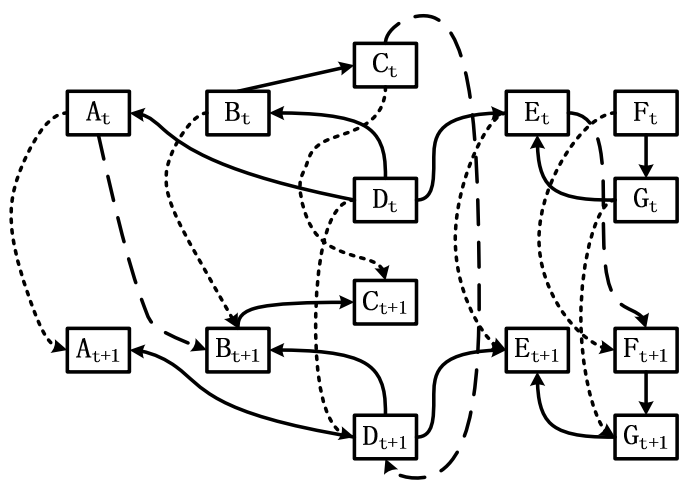

Figure 7. the ACM for model in fig.6

Notice that sometimes only unfold in time the pair of vertices disconnected by edges in MECS can lower the complexity of ACM efficiently. In fig 4.5 there is a node $G_{t}$, whose adjacent edges are not in MECS, this simplified ACM is only short of one node $G_{t+1}$ and one edge $G_{t} \rightarrow G_{t+1}$ than its original ACM.

Here step3.2 in algorithm 3 is applied to acquire MG of ACM, which is shown in fig.8. The dashed lines indicate the edges added, and the added edges are used to demonstrate morality of the directed graph. 


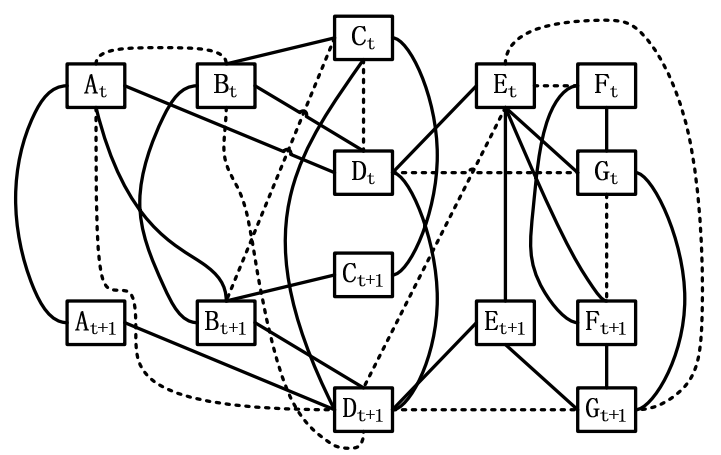

Figure 8. MG of the ACM in fig.7

Step 3.3 of algorithm 3 is preceded by considering edges in MG as the node to build corresponding HG, take $A_{t}$ and $A_{t+1}$ as example to demonstrate the hyper edge calculation:

$$
\begin{aligned}
& h_{A_{t}}=\left\{A_{t} \rightarrow A_{t+1}, A_{t} \rightarrow B_{t}, A_{t} \rightarrow B_{t+1}, A_{t} \rightarrow D_{t+1}\right\} \\
& h_{A_{t+1}}=\left\{A_{t}-A_{t+1}, A_{t+1} \rightarrow D_{t+1}\right\}
\end{aligned}
$$

Notice that two hyper edges can share nodes(edges) in HG(MG), like hyper edge $h_{A}$ and $h_{A+1}$ share the node $A_{t} \rightarrow$ $A_{t+1}$ in HG. Constant $c$ is chosen to equals $1 \%$ and $k$ equals 2 , step 3.4 in algorithm 3 then be performed to acquire edge cut set of HG as follow:

$$
\left\{h_{A_{t}}, h_{B_{t}}, h_{C_{t}}, h_{D_{t}}, h_{E_{t}}, h_{D_{t+1}}, h_{E_{t+1}}, h_{G_{t+1}}\right\}
$$

It follows that the node set of MG is $\left\{A_{t}, B_{t}, C_{t}, D_{t}, E_{t}, D_{t+1}, E_{t+1}, G_{t+1}\right\}$, the two regions of the partition is $\left\{A_{t+1}, B_{t+1}, C_{t+1}\right\}$ and $\left\{F_{t}, G_{t}, F_{t+1}\right\}$, which is shown in fig.9

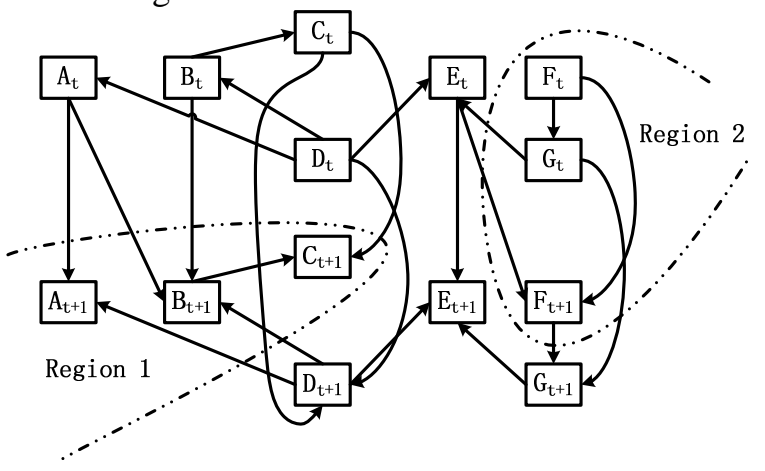

Figure 9. causally independent partition of ACM in fig.7

\section{F. Improved partition algorithm and its application in electric traction system}

Notice that though the digraph is divided into 2 regions and the partition can represent the original system feather. But 8 out of 14 nodes are on the border, the large size of border has no advantages in decrease of diagnosis complexity. So minimal d-separation sets method is introduced to refine the proposed algorithm.

Algorithm 4: Improved partition algorithm

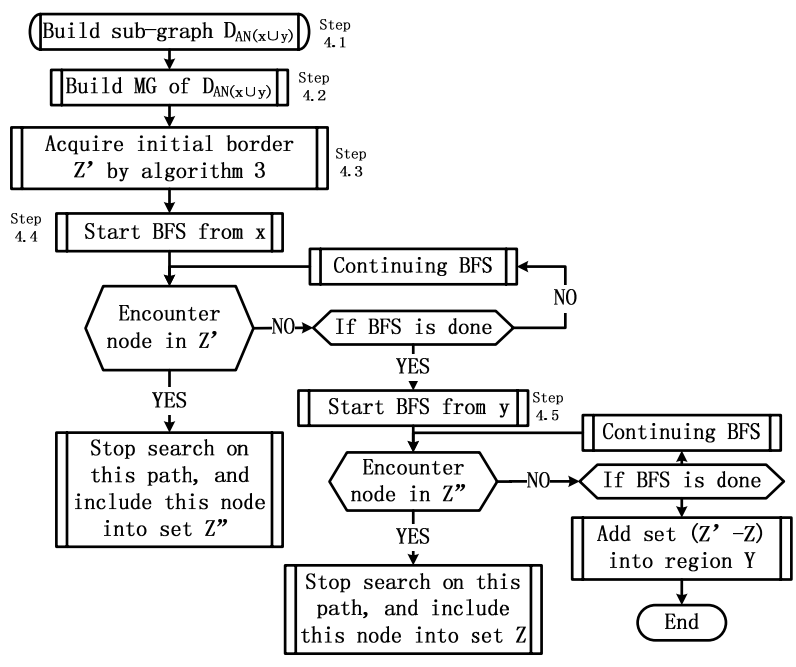

Figure 10. procedure of algorithm 4

In step 4.1, sub-graph $D_{A n(x \cup y)}$ indicates the ancestors of $x$ and $\mathrm{y}$. If there is a node $\mathrm{w}$ is connected to another node $\mathrm{u}$ through a directed path, then $\mathrm{w}$ is called ancestor of node $u$, if there is a node set $\left\{\mathrm{v}_{1}, \mathrm{v}_{2}, \ldots, \mathrm{v}_{\mathrm{n}}\right\}, \mathrm{w}=\mathrm{v}_{1}, \mathrm{u}=\mathrm{v}_{\mathrm{n}}$, and $\left(\mathrm{v}_{\mathrm{i}}, \mathrm{v}_{\mathrm{i}+1}\right), \mathrm{i}=1, \ldots, \mathrm{n}-1$, this node set is a directed path in $\mathrm{D}_{\mathrm{AN}(\mathrm{x} \cup \mathrm{y})}$ 。

Algorithm 3 is valid on two disjoint sets of nodes $\mathrm{X}$ and $Y$. the improvement of algorithm 4 is two extra nodes $\mathrm{X}$ ' and $y^{\prime}$ introduced into $\left(D_{\mathrm{AN}(\mathrm{X} \cup \mathrm{Y})}\right)^{\mathrm{m}}$, and ensure that all nodes in $\mathrm{x}^{\prime}\left(\mathrm{y}^{\prime}\right)$ and $\mathrm{X}(\mathrm{Y})$ are surely connected. Thus the goal of independent partition is transferred into finding the separator of x' and y'. Algorithm 4 aims on finding a separator set $Z$, the separator set of $\mathrm{x}$ and $\mathrm{y}$ in MG can be a good candidate, and it will d-separate $\mathrm{x}$ and $\mathrm{y}$ in the original directed graph $\mathrm{D}$.

The border obtained in algorithm 3 is defined as $Z^{\prime}$ in algorithm 4. Let $X=\left\{A_{t+1}, B_{t+1}, C_{t+1}\right\}, Y=\left\{F_{t}, G_{t}, F_{t+1}\right\}$, the BFS tree started at node $\mathrm{x}$, which is added into MG on step 4.4 algorithm 4, is shown in fig. 11.

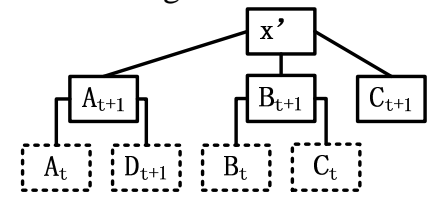

Figure 11. BFS tree in step 4.4 algorithm 4

The BFS result Z" is $\left\{A_{t}, B_{t}, C_{t}, D_{t+1}\right\}$, which is shown in fig.11 as the dashed part. The BFS tree started at node $\mathrm{y}$, which is added into MG on step 4.5 algorithm 4, is shown in fig.12. Here, Z= Z", which is shown in fig.12 as the dashed part.

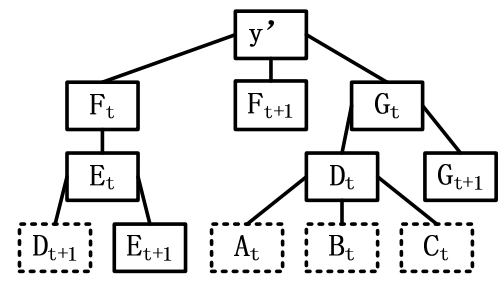

Figure 12. BFS tree in step 4.5 algorithm 4 
After step 4.5, add set ( $Z^{\prime}-Z$ ) into region $Y, Z$ will dseparate $\mathrm{X}$ and $\mathrm{Y}$. the new partition is shown in fig 4.11 . There are only 4 nodes in the border, which is half the size compares to the result of algorithm 3. Thus the diagnosis complexity is simplified significantly. The new partition can be further partitioning using algorithm 3 .

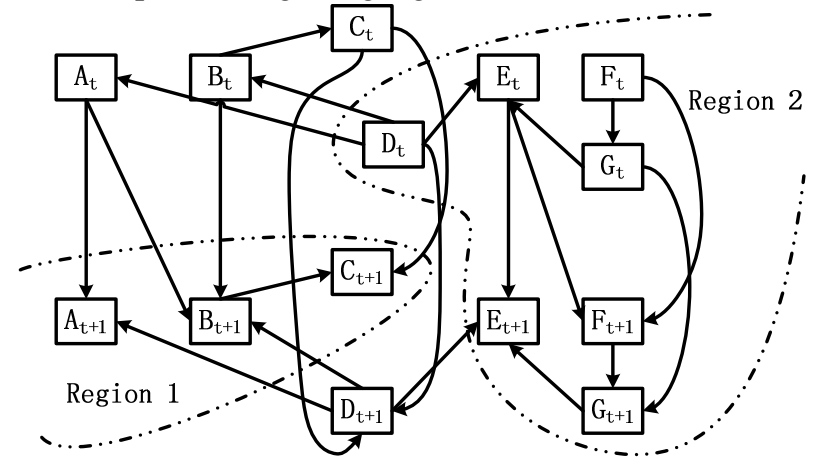

Figure 13. the optimized partition obtained by Algorithm 4

The main feathers of the partition obtained by algorithm 4 are that (1) the nodes number on border can be certain; (2) each region is causally independent from the other region in this partition. From the fault diagnosis perspective, diagnosis for each region can be performed locally, and all the information need to be known is the state of nodes inside this region and on the border. Thus, the electric traction system diagnosis system is designed into 2 regions according to the upper analyze.

\section{FAUlT DiAgNOSIS SYSTEM DESIGN}

\section{A. Prototype design}

The engineering of distributed fault diagnosis system of electric locomotive electric traction system is currently still at a beginning stage in China. Status monitoring and fault diagnosing are for single device instead of systems. On the basis of the theory of this paper, several researches on engineering of fault diagnosis have been done to design system-level status motoring and fault diagnosis system of electric traction system. Fig.14 shows the basic diagram.

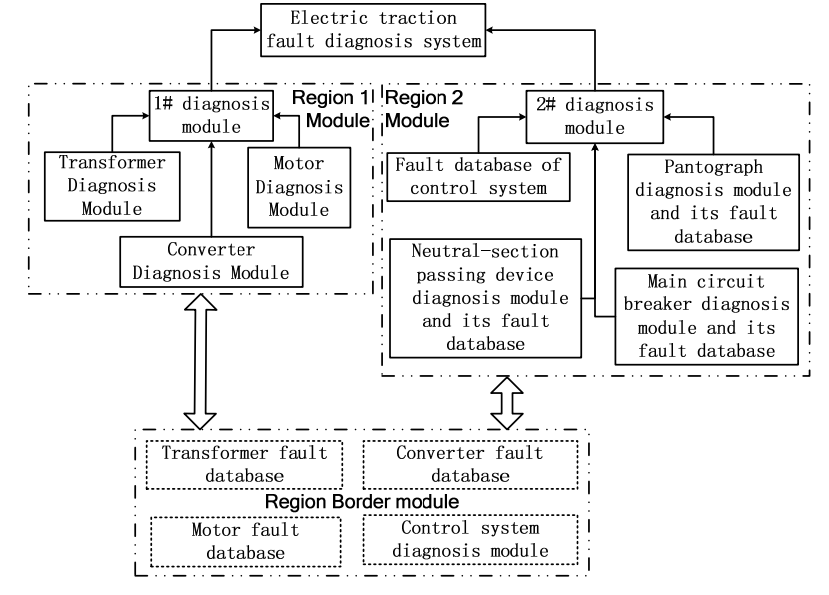

Figure 14. diagnosis scheme of electric traction system
Based on the analysis in the last three sections, the fault diagnosis system for electric traction system is divided into three areas as showed in fig.14. The $1 \#$ and 2\# diagnosis module in fig. 14 is corresponded to region 1 and region 2 in system causal model partition. The region border diagnosis module is mainly composed of measuring database and control system diagnosis sub-module. The region border diagnosis module works only when there are nodes in $1 \#$ and 2\# diagnosis module had unreliable measures, which can be interpreted as the region independent is compromised. When nodes in 1\# (2\#) diagnosis module fail, the border diagnosis module communicates with $1 \#$ (2\#) diagnosis module to compensate the causality independence.

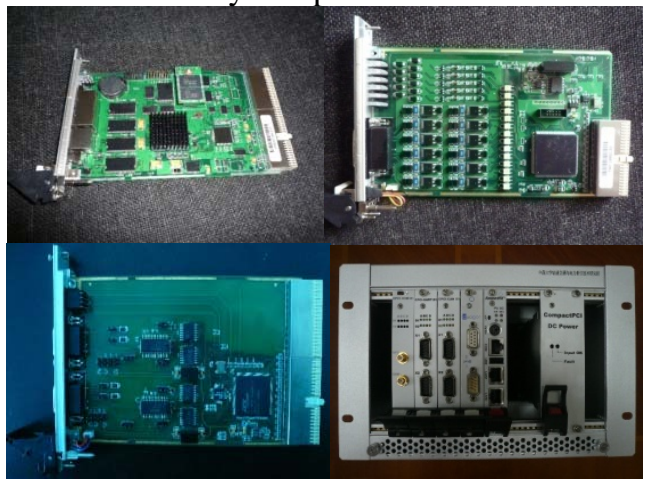

Figure 15. diagnosis system prototype

According to the analysis above, a Pentium IV CPU is adopted as main processor of the diagnosis system to ensure real-time performance; compact-PCI bus is selected as the system bus. Fig.15 shows the CPU board, Communication board, $\mathrm{I} / \mathrm{O}$ board and the system cabinet. The diagnosis of the whole electric traction system is simplified into locally diagnosis of different regions, the measures and computational complexity is reduced, the diagnosis speed and efficiency is enhanced, so the online monitoring of the whole electric traction system become possible. With regard to the fault caused by subsystem coupling, the proposed diagnosis system can diagnosis from a system perspective, and can achieve a better accuracy. Moreover different optimized diagnosis method which suits the best for each subsystem can be implemented in each region to reach the best diagnosis effect.

\section{B. Experimental data}

There are 3 sets of prototype applied to the field. After a year, the 3 prototype has diagnosis 54 faults in various kinds, among which, there are 7 faults are caused by multiple coupled sources.

Table III shows the average annual failure frequency given by each fault diagnosis module and the actual fault frequency. The comprehensive fault diagnostic accuracy is $96.4 \%$, and is acceptable. But there are several unsolved issue that (1) some of the sub-fault diagnosis module has lower diagnostic accuracy due to the drawback of its local diagnosis method; (2) the running time of the prototype is not long enough, there is a certain randomness in its diagnostic accuracy data. 
TABLE III. AVERAGE ANNUAL FAILURE FREQUENCY COMPARISON

\begin{tabular}{c|c|c|c|c}
\hline No. & Subsystem & $\begin{array}{c}\text { diagnosis } \\
\text { fault }\end{array}$ & $\begin{array}{c}\text { Actual } \\
\text { fault }\end{array}$ & $\begin{array}{c}\text { diagnostic } \\
\text { accuracy rate }\end{array}$ \\
\hline 1 & Transformer & 1.67 & 1.33 & $74.4 \%$ \\
\hline 2 & Converter & 3 & 3.33 & $90.1 \%$ \\
\hline 3 & Motor & 3.67 & 3.33 & $89.8 \%$ \\
\hline 4 & Control & 5.33 & 5.67 & $94 \%$ \\
\hline 5 & $\begin{array}{c}\text { Neutral-section } \\
\text { passing device }\end{array}$ & 0.67 & 0.67 & $100 \%$ \\
\hline 6 & Pantograph & 1 & 1.33 & $75 \%$ \\
\hline 7 & $\begin{array}{c}\text { Main circuit } \\
\text { breaker }\end{array}$ & 2.67 & 3 & $89 \%$ \\
\hline & Total & 18 & 18.67 & $96.4 \%$
\end{tabular}

\section{CONCLUSION}

The practicality of distributed fault diagnosis 分 system on electric traction system of locomotives is proved by tests. Although the accuracy and reliability of system diagnosis is practical, there is still room for further improvement. For instance, the method of partial diagnosis needs to be improved: the method of pattern recognition analyzes more accurately based on signals and fault characteristics. The parameters of fault diagnose needs to be expanded by adding measures such as vibration, audio and Image by information fusion technology to diagnose more completely and accurately.

\section{ACKNOWLEDGMENT}

This work is supported by National High Technology Research and Development Program of China (No.2011AA110505).

\section{REFERENCES}

[1] Cheng Shu, Chen Te-fang, Yu Ming-yang. An Novel FB-ZVZCS PWM Converter Using an Improved secondary Active Clamping Circuit. Proceedings of the CSEE.2008.28(12):44-50

[2] Liwei, Chen Te_fang, Cheng Shu. Research on Multi-Sensors Distributed Fault Diagnosis Theory of Locomotive Electric System. Journal of the China Railway Society.2010.5:70-76

[3] Fabre E, Benveniste A, Jard C. Distributed diagnosis for large discrete events in dynamic systems. In: Proceedings of the 15th IFAC World Congress, Barcelona, Spain. 2002

[4] Albert M, Längle T, Wörn H, Kazi A, Brighenti A, Senior C, Revuelta Seijo C,Sanz Bobi MA, Villar J Distributed architecture for monitoring and diagnosis. EU ESPRIT Project DIAMOND.2001

[5] Letia A, Craciun F, Kope Z, Netin A. Distributed diagnosis by BDI agents. Proceedings of the OASTED International Conference on Applied Informatics, 2000

[6] Köpen-Seliger B, Marcu T, Capobianco M, Gentil S, Albert M, Latzel S. MAGIC: An integrated approach for diagnostic data managemen and operator support. In: Proceedings of the IFAC Symposium SAFEPROCESS'03, Washington, USA, 187-192. 2003

[7] Isermann R, Ballé P. Trends in the application of model-based fault detection and diagnosis of technical processes. Control Engineering Practice. 5(5):709-719. 1997

[8] Bocaniala CD,Sa da Costa J. Novel framework for using causal models in distributed fault diagnosis[C]. Proceedings of Workshop on Advances in Control and Diagnosis. Karlsruhe,Germany. 2004:142 147

[9] Bocaniala CD,Sa da Costa J. Novel methodology for partitioning complex systems for fault diagnosis purposes[C]. Proceedings of the 16th IFAC.2005 World Congress,Praha, Czech Republic.2005

[10] Su Ran, Zhang Lie-yong,Gu Xue-ping. Distributed Fault Diagnosis of Power Networks Applying the United Rules Mining Algorithm Based on Rough Set Theory. Proceedings of the CSEE.2010.30(4):28-34 